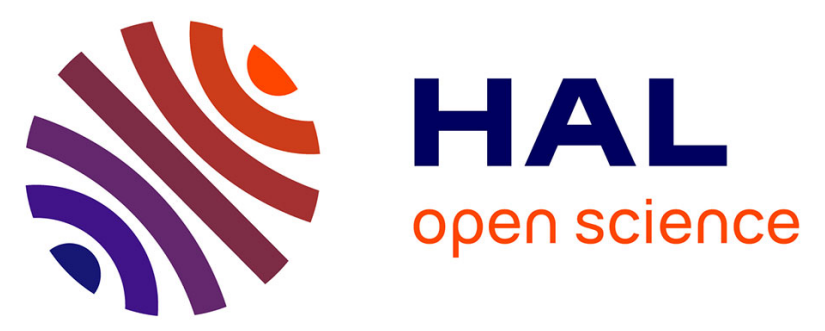

\title{
An automatic approach towards modal parameter estimation for high-rise buildings of multicomponent signals under ambient excitations via filter-free Random Decrement Technique
}

\author{
Fatima Nasser, Zhongyang Li, Nadine Martin, Philippe Gueguen
}

\section{To cite this version:}

Fatima Nasser, Zhongyang Li, Nadine Martin, Philippe Gueguen. An automatic approach towards modal parameter estimation for high-rise buildings of multicomponent signals under ambient excitations via filter-free Random Decrement Technique. Mechanical Systems and Signal Processing, 2016, 70-71, pp.821-831. 10.1016/j.ymssp.2015.08.008 . hal-01233152

\author{
HAL Id: hal-01233152 \\ https://hal.science/hal-01233152
}

Submitted on 24 Nov 2015

HAL is a multi-disciplinary open access archive for the deposit and dissemination of scientific research documents, whether they are published or not. The documents may come from teaching and research institutions in France or abroad, or from public or private research centers.
L'archive ouverte pluridisciplinaire HAL, est destinée au dépôt et à la diffusion de documents scientifiques de niveau recherche, publiés ou non, émanant des établissements d'enseignement et de recherche français ou étrangers, des laboratoires publics ou privés. 


\title{
An Automatic Approach towards Modal Parameter Estimation for High-Rise Buildings of Multicomponent Signals under Ambient Excitations via Filter-free Random Decrement Technique
}

\author{
Fatima Nasser ${ }^{\mathrm{a}}$, Zhongyang $\mathrm{Li}^{\mathrm{a}}$, Nadine Martin ${ }^{\mathrm{a}, *}$, Philippe Gueguen ${ }^{\mathrm{b}}$ \\ ${ }^{a}$ Univ. Grenoble Alpes, GIPSA-Lab, F-38000 Grenoble, France \\ CNRS, GIPSA-Lab, F-38000 Grenoble, France \\ ${ }^{b}$ ISTerre, Univ. Grenoble /CNRS/IFSTTAR \\ BP 53, 38041 Grenoble cedex 9, France
}

\begin{abstract}
This paper proposes an automatic modal analysis approach for signals of highrise buildings recorded under real-world ambient excitations. The fact of working over such type of signals is faced with several challenges: the time-domain convolution between the system impulse response and the seismic noise, the existence of several components, the presence of closely-spaced frequency modes, with high additive noises, and low, exponential and damped amplitudes. The proposed approach handles these challenges simultaneously without the need for a user intervention. It is based on a filter-free Random Decrement Technique to estimate the free-decay response, followed by a spectral-based method for a rough modal estimate and finalized by a Maximum-Likelihood Estimation process to refine the modal estimates. Each of these processes is responsible to tackle one or more of the aforementioned challenges in the aim to provide an automatic and moreover a reliable modal analysis of the studied signals.
\end{abstract}

Keywords: Automatic modal analysis, ambient vibrations, Random Decrement Technique, damping ratios, closely-spaced frequency.

\footnotetext{
${ }^{*}$ Corresponding author

Email addresses: fatima.nasser@gipsa-lab.grenoble-inp.fr (Fatima Nasser), zhong-yang.li@gipsa-lab.grenoble-inp.fr (Zhongyang Li),

nadine.martin@gipsa-lab.grenoble-inp.fr (Nadine Martin),

Pilippe.gueguen@obs.ujf-grenoble.fr (Philippe Gueguen)
}

Preprint submitted to Journal of Mechanical Systems and Signal ProcessingNovember 24, 2015 


\section{Introduction}

As the need for continuous monitoring of a building health is increasing, so does the need for automatic modal analysis techniques. Such techniques can assess the structural health in a passive way without resorting to a manual user

In this regard, several automatic techniques of output-only modal identification have been proposed in the last decade. The first proposal can be found in [1] and is based on the least square complex frequency method [2]. A stochastic subspace identification (SSI) method has been proposed in [3] and has been applied to monitor the dynamic behavior of a Bridge in Switzerland, and to track the modal changes versus the environmental conditions of a bridge in England [4]. [5] has proposed an automated modal analysis procedure based on SSI as well. Such a proposition relied on the selection of physical poles on a stabilization diagram which precludes the full automation of the modal analysis It is based either on a frequency domain technique including specific indicators for the automation, or on an SSI multipath merging technique which provides a simple stabilization diagram analyzed with an alignment criterion. Porto university [7, 8, 9] has proposed another automated approach based on a covariance A partially automated method based on a Frequency Domain Decomposition (FDD) approach for multi-channel monitoring system has been also proposed in Porto university. [10] has proposed an automated method based on a blind source separation method applied on a set of time series. An automated FDD is

[12 an algorithm is developed for fully automated operational modal analysis [13] proposed a mode clustering as a preliminary step for performing a physical mode selection. 
However, the existing methods have certain limitations that can be summa-

30 higher frequency, highly damped, poorly excited and closely-spaced frequency modes difficult to be identified. (2) Relies on a threshold-based peak detection, at which a preliminary calibration process is required. Such a calibration gets worse in the presence of noisy measurements which results in false-alarmed peakmore than one sensor to be used.

The work presented in this paper can be thought of as a contribution to avoid the aforementioned limitations of the existing methods. First, we propose a filter-free random decrement technique to automatically estimate a free-decay 40 response. Second, we propose a non-parametric spectral-based method to automatically identify the number of modes in the estimated free-decay response and to estimate the associated modal parameters of each of the identified modes. Finally, we propose a parametric method based on a maximum-likelihood estimator to refine the modal parameter estimation and provide more reliable and damped ones.

These steps make the proposed approach of this paper applicable over a single-channel record of multi-mode signals despite of the noise level, furthermore through the use of the statistical properties of the estimator, the local peaks of the multi-mode signal are detected by a detection method which is insensitive to the settings.

The remainder of this paper is organized as follows: Section 2 presents the Filter-Free Random Decrement Technique and defines the output model as a Multi-mode Random Decrement Signature. Section 3 derives an original nonparametric approach for detecting the number of modes and estimating the modal parameters. In section 4 a Maximum-Likelihood Estimation is proposed for refining the modal parameter estimation. Section 5 presents the results of the proposed AMBA over six simulated signals. Finally, conclusions are drawn in section 6 


\section{Filter-free Random Decrement Technique}

When aiming to estimate the modal parameters, one first important step to take into consideration is the estimation of the free-decay response. In this context, two estimation family methods exist in the literature, one stems from the correlation function of the ambient response [14, 15, the second one from the well-known Random Decrement Technique (RDT) [16, 17]. Both ways of estimate need the assumption of a white noise for the ambient vibration and have advantages and drawbacks due to different estimation processes. In the two cases when dealing with multi-component signals which is often the case for actual buildings, we need then either to pre-filter the signal, or to propose 70 a way of separating the different modes directly from the free decay response. For example, [15] proposed to apply a time-scale transform and then to extract the instantaneous frequencies of the different modes by a ridge method. The limitations of this approach are the wavelet choice and the Fourier resolution inherent to a non-parametric time-frequency approach.

In this paper, we focus on the RDT for the estimation of the free-decay response. It is a method proposed by Cole [18] in 1968 as an efficient method to extract a free-decay signature equivalent to the system impulse response that is denoted as RDS. It was first used by Ibrahim [19] in 1977 for modal identification. Then Vandiver et al. in 1982 [20] derived its mathematical basis. In the context of our work, to tackle the challenge of the time-domain convolution between the impulse response and the seismic noise, we proposed to first use the RDT.

When the RDT was first proposed by Cole, it was not directly applied to a multi-component time series of the structural response, but was only based on a Single Degree of Freedom (SDOF) system [18]. The data was necessarily filtered prior to the RDT application (c.f Fig. 1).

However, it is well-known that the filtering process is a critical step especially in situations where data are difficult to be analyzed, i.e., low signal to noise ratio, closely-spaced frequency modes, heavily damped modes, etc., which are 


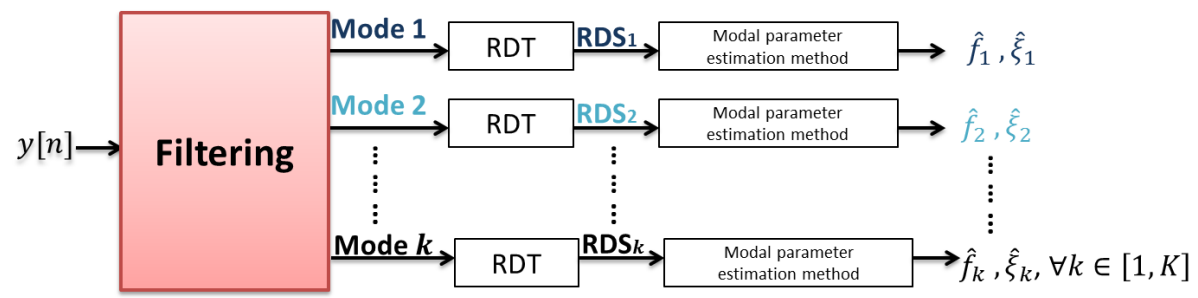

Figure 1: The general concept of the Random Decrement Technique.

the characteristics of almost all the real-world signals that generally have a large number of modes.

Accordingly, filtering is one of the very delicate steps when dealing with the RDT. Tuning the filtering process manually is very time consuming, and totally dependent on the user.

As the main objective of this work is to automate the estimation process of the modal parameters using the RDT, this paper thus proposed an approach which skips this preliminary filtering. Such a procedure is referred to as a Filter-Free Random Decrement Technique that leads to the estimation of the Multi-mode Random Decrement Signature (MRDS) (Fig. 2).

The originality of the Filter-Free RDT is its ability to: (1) avoid the difficulty encountered in the filtering process especially in situations where data are difficult to be analyzed, i.e., low signal-to-noise ratio levels, closely-spaced frequency modes, and heavily damped modes, and (2) skip the required user intervention that definitely precludes the automatic estimation procedure.

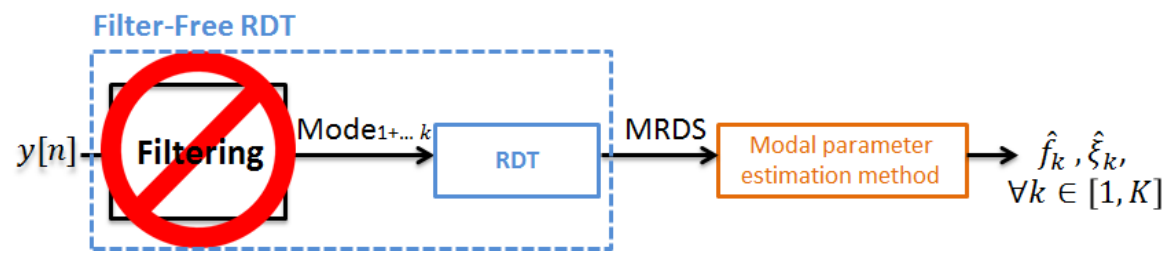

Figure 2: Illustration of the Filter-Free Random Decrement Technique.

An MRDS is characterized by the same number of modes $K$, the same damping ratio $\xi_{k}$ and natural frequency $f_{k}$ as the impulse response $h[n]$ of the mea- 
sured building vibration $y[n]$, but not the initial phase and the initial amplitude, as these two parameters are changed due to the averaging principle of the RDT.

The measured building vibration $y[n]$ is given as in [21]

$$
\begin{gathered}
\qquad y[n]=\mathfrak{g}[\mathfrak{n}]+e[n], \\
\text { where } \mathfrak{g}[\mathfrak{n}]=h[n] * p[n] \\
\text { with } h[n]=\sum_{k=1}^{K} A_{0 k} \exp ^{-2 \pi f_{k} \xi_{k} n} \sin \left(\omega_{D k} n+\phi_{0 k}\right), \forall k \in[1, K],
\end{gathered}
$$

where $n$ is the normalized discrete time index, $\mathfrak{g}[\mathfrak{n}]$ is the noise-free part of the building vibration, $h[n]$ is the impulse response of a building. The initial amplitude $\left(A_{0 k}=-1 / \omega_{D k}\right)$, the damping ratio $\left(\xi_{k}\right)$, the natural frequency $\left(f_{k}\right)$, the initial phase $\left(\phi_{0 k}\right)$, and the number of modes $(K)$ are the parameters that feature $h[n] . \omega_{D k}=2 \pi f_{k} \sqrt{1-\xi_{k}^{2}}$ is the damped pseudo-pulsation, $p[n]$ and $e[n]$ are the seismic and the additive noises respectively, both assumed to be white Gaussian of zero mean and unknown variance. The convolution operator is $*$.

Considering all the above, in this paper we proposed a signal model for the estimated MRDS $s[n]$ as

$$
s[n]=\mathfrak{h}[\mathfrak{n}]+v[n],
$$

$$
\text { with } \quad \mathfrak{h}[\mathfrak{n}]=\sum_{k=1}^{K} B_{0 k} \exp ^{-2 \pi f_{k} \xi_{k} n} \sin \left(2 \pi f_{k} n+\varphi_{0 k}\right), \forall k \in[1, K],
$$

where $v[n]$ is the residue of the RDT considered as an additive white Gaussian noise with zero mean and unknown variance. $\mathfrak{h}[\mathfrak{n}]$ is a deterministic multi-mode process that keeps the same characteristics as $h[n]$ of Eq. (2) but not for the initial phase $\varphi_{0 k}$ and the initial amplitude $B_{0 k}$. It should be noted that the term $\sqrt{1-\xi_{k}^{2}}$ of Eq. (2) is approximately equal to 1 in the considered signal model due to the fact that the range of the damping ratio in the real-world cases is rarely greater than $10 \%$.

In this paper, this signal model is used for deriving a maximum likelihood 
approach in order to refine the modal parameter estimates so as to meet the above cited constraints.

\section{Preliminary estimation of the modal parameters}

The issue is now to handle the presence of multi-modes in the estimated MRDS, at which for each mode a natural frequency and a damping ratio are to be estimated. For this purpose, we proposed a spectral-based method based on the Welch spectral estimation of the MRDS. Furthermore, we proposed to include a peak-detection method, namely the one proposed by Durnerin [22], to automatically detect the number of modes in the MRDS.

\subsection{Hypothesis Test for Peak Detection and Removal}

The spectrum of $\mathrm{s}[\mathrm{n}]$ of Eq. (3) can be expressed as

$$
\mathbf{S}_{s}[f]=\mathbf{S}_{\mathfrak{h}}[f]+\mathbf{S}_{v}[f]
$$

135 where $\mathbf{S}_{\mathfrak{h}}[f]$ is the spectrum of the noise-free part $\mathfrak{h}[\mathfrak{n}]$, and $\mathbf{S}_{v}[f]$ is the spectrum of the random part $v[n]$. The purpose of the detector is to decide for each frequency $f$ of the spectrum $\mathbf{S}_{s}[f]$ whether $s[n]$ consists of only noise $v[n]$, or the sum of $\mathfrak{h}[\mathfrak{n}]$ and the noise $v[n]$. For that purpose, a statistical test between the two hypotheses can be written as in 23 .

$$
T[f]=\frac{\mathbf{S}_{s}[f]}{\mathbf{S}_{v}[f]} \underset{\mathcal{H}_{0}}{\gtrless} \underset{\mathcal{H}_{1}}{\gtrless},
$$

with $T[f]$ a random variable, $\lambda$ the detection threshold, and the two hypotheses deduced from Eq. 5 being defined as

$$
\begin{aligned}
& \mathcal{H}_{0}: \mathbf{S}_{s}[f]=\mathbf{S}_{v}[f] \\
& \mathcal{H}_{1}: \mathbf{S}_{s}[f]=\mathbf{S}_{\mathfrak{h}}[f]+\mathbf{S}_{v}[f]
\end{aligned}
$$


In this study, the probability density of $\mathcal{H}_{1}$ is unknown, so the spectral peak detector as proposed in [22] is applied. Such a detection is based on the NeymanPearson test where the probability of false alarm $\mathcal{P} \mathcal{F} \mathcal{A}$ is given a priori to be able to fix the threshold $\lambda$ such as

$$
\mathcal{P F} \mathcal{A}=\int_{\lambda}^{+\infty} p_{T(f) \mid \mathcal{H}_{0}}(x) d x,
$$

$\mathcal{P F} \mathcal{A}$ is likely to retain $\mathcal{H}_{1}$ while $\mathcal{H}_{0}$ is true. $p_{T(f) \mid \mathcal{H}_{0}}=\chi_{2}^{r}$ is the probability density of $T(f)$ under the hypothesis $\mathcal{H}_{0}$. Indeed, it has been shown in [22] that all the Fourier estimators under the hypothesis $\mathcal{H}_{0}$ can be considered proportional to a random variable following a $\chi_{2}^{r}$ distribution of degree of freedom $r$, with $r$ being dependent on the number of segments and their overlapping.

The noise spectrum $\mathbf{S}_{v}[f]$ of Eq. (5) is unknown and therefore must be estimated in order to apply Eq. (6) for detecting the modes in the spectrum.

\subsection{Noise Spectrum Estimation}

Two types of errors can be encountered in the estimation of the noise spectrum: the underestimation and the overestimation. In the first case, some of the modes can be wrongly identified as noise. In the second case, noise peaks could be considered as modes and cause false alarms. These two errors should be avoided in order to ensure the robustness of the approach.

The unknown noise spectrum $\mathbf{S}_{v}[f]$ of Eq. 5 is proposed to be approximated as $\hat{\mathbf{S}}_{v}[f]$ through an iterative process; namely, the multipass filtering technique developed in 22], and shown in Fig. 3

This technique is initialized with a median filter

$$
\hat{\mathbf{S}}_{v 1}[f]=F I L T_{\text {med }}\left\{\mathbf{S}_{s}\left[f-\frac{\left(L_{f}-1\right)}{2}, \cdots, f+\frac{\left(L_{f}-1\right)}{2}\right]\right\},
$$

where $\hat{\mathbf{S}}_{v 1}[f]$ is the noise estimation of the first iteration, $F I L T_{\text {med }}\{\cdot\}$ is the median filter, and $L_{f}$ is an odd integer specifying the length of the sliding filter 


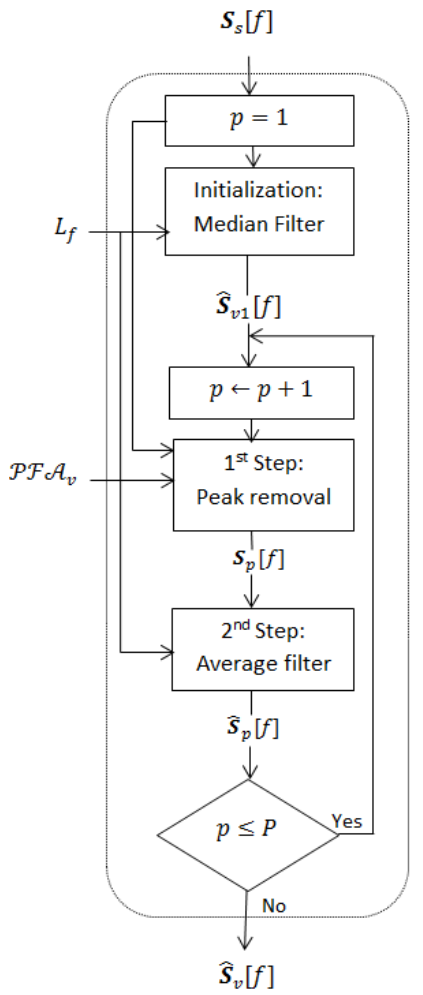

Figure 3: An overall flowchart of the noise spectrum estimation process.

window. Normally $L_{f}$ is defined as

$$
3 B_{\text {main }}\left(\frac{F_{s}}{f_{\text {res }}}\right) \leq L_{f} \leq 4 B_{\text {main }}\left(\frac{F_{s}}{f_{\text {res }}}\right),
$$
the chosen window function, for Hamming window $B_{\text {main }}=4 . F_{s}$ the sampling frequency of the signal, and $f_{r e s}$ the frequency resolution of the spectrum.

The method comprises $P$ iterations of nonlinear filtering. The noise spectrum estimate is refined at each iteration $\forall p \in[1, P]$ by suppressing the peaks belonging to the spectrum $\mathbf{S}_{p}[f]$. The refined spectrum is then recorded $\hat{\mathbf{S}}_{p}[f]$.

Each following iteration has two steps. In the first step, the peaks corresponding to $\mathcal{H}_{1}$ are removed by applying the hypothesis test described in section 3.1 with a probability of false alarm referred to as $\mathcal{P} \mathcal{F} \mathcal{A}_{v}$ and the current 
estimated noise spectrum $\hat{\mathbf{S}}_{p-1}[f]$. The second step smooths the remaining part $\mathbf{S}_{p}[f]$ by an average filter with a sliding window of $M$ points. The final noise spectrum estimation $\hat{\mathbf{S}}_{v}[f]$ is the output after $P$ iterations. The choice of the number of $P$ is set to 3 to 5 iterations since experimentally the noise spectrum stops changing after such a range.

\subsection{Mode detection and frequency estimation}

As soon as the noise spectrum is estimated, the peaks of the spectrum $\mathbf{S}_{s}[f]$ can be calculated using Eq. (6) where

1- $\lambda$ in Eq. (6) is deduced from a probability of false alarm $\mathcal{P F} \mathcal{A}_{d}$ as in Eq. (8).

2- $\mathbf{S}_{v}[f]$ in Eq. (6) is substituted using the estimated noise spectrum $\hat{\mathbf{S}}_{v}[f]$ of section 3.2 .

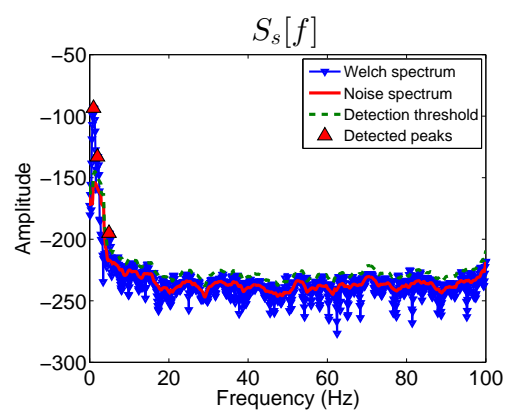

(a)

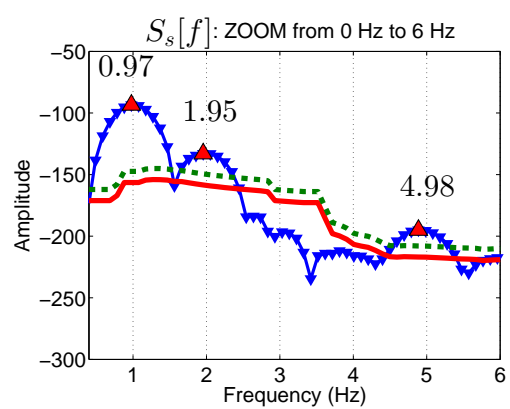

(b)

Figure 4: (a) Application of the peak detection method on the Welch spectrum of a simulated signal with $f=1,2$ and $5 \mathrm{~Hz}$ and $\xi=1,2$ and $3 \%$ respectively, (b) a zoom on the three detected modes.

The peaks are defined as the vertex of the bell shapes above the detection threshold $\lambda$. The number of modes $K$ of $h[n]$ of Eq. 2 are estimated as the number of the detected peaks. In [22] a spectral window matching technique is used to estimate the frequency of the peak; however, in this study, since only rough estimates are desired in the preliminary estimation of the modal 
parameters procedure, the maximum of the bell shape is defined to be the position $f$ of each peak which defines the frequency estimation of each of the associated peaks.

The peak detection is illustrated in Fig. 4 with a simulated signal of 180000 points length, sampled at $200 \mathrm{~Hz}$, consists of three modes located at $1 \mathrm{~Hz}, 2 \mathrm{~Hz}$, and $5 \mathrm{~Hz}$ and damped by $1 \%, 2 \%$ and $3 \%$ respectively. The detection threshold is calculated with a probability of false alarm $\mathcal{P} \mathcal{F} \mathcal{A}_{v}=0.1$, and another probability of false alarm of the noise spectrum estimation $\mathcal{P} \mathcal{F} \mathcal{A}_{d}=0.1$.

Fig. 4 presents a proper functionality of the mode detection and frequency estimation procedure. The three modes of interest are being correctly identified with reasonable frequency location as compared with the theoretical ones, with a normalized error $e_{\hat{f}^{\prime}}=\left|f-\hat{f}^{\prime}\right| / f \leq 0.03 \%$.

\subsection{Damping Ratio Estimation}

Hereby, we address the issue of estimating the damping ratio for all the estimated modes of the MRDS. In this paper, the estimation is achieved using the ratio of the spectra of two neighboring segments over $s[n]$. These two segments are of the same length $L_{t}=2 / 3 L_{r d s}$ and placed one at the beginning of the MRDS and the other at its end. They have an overlapping of 50\% (Fig. 5 (a)).

The periodogram on each segment is calculated using a Hamming window to have more distinct frequency representation of the mode. The two applied windows are defined as $\mathbf{W}_{1}=\left[0,2 / 3 L_{r d s}\right]$ and $\mathbf{W}_{2}=\left[2 / 3 L_{r d s}, L_{r d s}\right]$ on the two segments respectively. The windowing of the two segments yields two periodograms $\mathbf{S}_{1}[f]$ (Fig. $5(\mathrm{~b})$ ) and $\mathbf{S}_{2}[f]$ (Fig. 5 (c)).

Hereinafter, each segment is expressed in the time-domain in a vector form 215 as

$$
\begin{gathered}
\mathbf{s}_{1}=\left[s[1], \cdots, s\left[\frac{2}{3} L_{r d s}\right]\right], \\
\left.\mathbf{s}_{2}=\left[s\left[1+\frac{1}{3} L_{r d s}\right], \cdots, L_{r d s}\right]\right],
\end{gathered}
$$




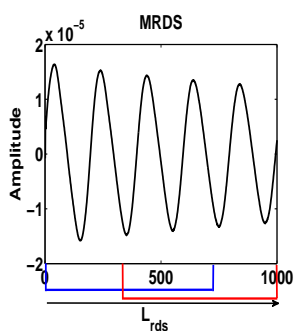

(a)

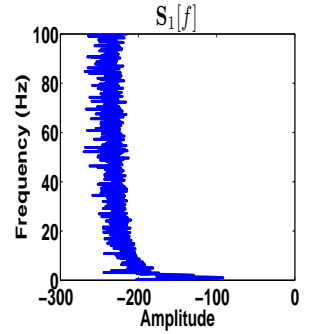

(b)

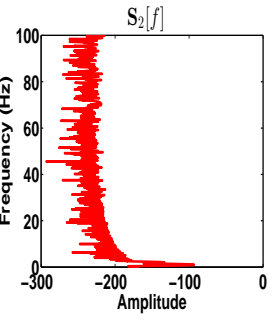

(c)

Figure 5: (a) An MRDS being split up into two segments of equal length with $50 \%$ overlapping, (b) and (c) the periodograms of the two segments respectively.

where $\mathbf{s}_{1}$ and $\mathbf{s}_{2}$ are the MRDS on the first and the second segment respectively.

Further on, $\mathbf{s}_{1}$ and $\mathbf{s}_{2}$ can be decomposed as the sum of modes

$$
\mathbf{s}_{j}=\sum_{k=1}^{K} \mathbf{s}_{j, k}, \forall k \in[1, K],
$$

where $j=1,2$ being an index to present the two vectors $\mathbf{s}_{1}$ and $\mathbf{s}_{2}$, and $\mathbf{s}_{1, k}=\left[s_{k}[1], \cdots, s_{k}\left[2 / 3 L_{r d s}\right]\right]$, and $s_{2, k}=\left[s_{k}\left[1+1 / 3 L_{r d s}\right], \cdots, s_{k}\left[L_{r d s}\right]\right]$ are the RDS vectors of the mode $k$ (Eq. (3p) over each segment.

For each segment $j$ we have

$$
\mathcal{F} \mathcal{T}\left\{\mathbf{s}_{j}\right\}=\sum_{k=1}^{K} \mathcal{S}_{j, k}[f]=\mathcal{S}_{j}[f] .
$$

At frequency $f_{k}$ with

$$
\mathcal{F} \mathcal{T}\left\{\mathbf{s}_{j, k}\right\}=\mathcal{S}_{j, k}[f]
$$

where $\mathcal{F} \mathcal{T}\{$.$\} denotes the Discrete Fourier Transform, and \mathcal{S}_{j, k}$ is the vector of the spectrum denoted as $\mathcal{S}_{j, k}[f]$.

Since if the power of the modes are independently distributed in the frequencydomain, then

$$
\mathcal{S}_{j}\left[f_{k}\right]=\mathcal{S}_{j, k}\left[f_{k}\right]
$$




$$
\frac{\mathcal{S}_{2}\left[f_{k}\right]}{\mathcal{S}_{1}\left[f_{k}\right]}=\frac{\mathcal{S}_{2, k}\left[f_{k}\right]}{\mathcal{S}_{1, k}\left[f_{k}\right]}
$$

If we assume that the noise in the MRDS is sufficiently reduced, then according to Eq. (3), the estimation of the peak amplitude becomes

$$
\frac{\mathcal{S}_{2}\left[f_{k}\right]}{\mathcal{S}_{1}\left[f_{k}\right]}=\exp ^{-\alpha \frac{1}{3} L_{r d s}}
$$

where $\alpha=2 \pi f_{k} \xi_{k}$, with $f_{k}$ and $\xi_{k}$ being the frequency and the damping ratio of the MRDS for each mode $k, \forall k \in[1, K]$. For each detected peak at the two time instants $1 / 3 L_{r d s}$ and $2 / 3 L_{r d s}$ we estimate the damping ratio as

$$
\hat{\xi_{k}^{\prime}}=\frac{\log \left(\mathcal{S}_{1}\left[f_{k}\right]\right)-\log \left(\mathcal{S}_{2}\left[f_{k}\right]\right)}{2 \pi f_{k} \frac{L_{r d s}}{3}} .
$$

\section{Refined estimation of the modal parameters}

Once the number of modes of the MRDS along with the associated modal parameters are estimated, we propose a refined parametric estimation method in order to improve the frequency resolution and to be able to better estimate the closely-spaced frequency modes as compared to the non-parametric approach. The method is based on a Maximum-Likelihood Estimation approach. This method fits well the challenge of closely-spaced frequency modes as well.

The parameters of each mode of Eq. (3) form a vector $\boldsymbol{\theta}$,

$$
\boldsymbol{\theta}=\left[\boldsymbol{\theta}_{1}^{T}, \cdots, \boldsymbol{\theta}_{k}^{T}\right]=\left[B_{0 k}, \xi_{k}, f_{k}, \varphi_{0 k}\right]^{T}, \forall k \in[1, K],
$$

where ${ }^{T}$ is the transpose symbol, and $B_{0 k}, \xi_{k}, f_{k}$ and $\varphi_{0 k}$ are the initial amplitude, the damping ratio, the natural frequency, and the initial phase of the $k^{t h}$ mode respectively.

The MLE of $\boldsymbol{\theta}$ is considered to be equivalent to the least square approach under the assumption of white Gaussian additive noise [24]. Thus it results in 
the following non-linear equation

$$
\begin{gathered}
\hat{\boldsymbol{\theta}}=\underset{\boldsymbol{\theta} \in \mathbb{N}^{4 k}}{\arg \max } l_{M L E}(\boldsymbol{\theta})=\underset{\boldsymbol{\theta} \in \mathbb{N}^{4 k}}{\arg \min } L S(\boldsymbol{\theta}), \\
L S(\boldsymbol{\theta})=\sum_{n=-L / 2}^{L / 2}|s[n]-\mathfrak{h}[\mathfrak{n} ; \boldsymbol{\theta}]|^{2} .
\end{gathered}
$$

${ }_{245} L S(\boldsymbol{\theta})$ is the least square function. $s[n]$ is the MRDS (Eq. (3)), $\mathfrak{h}[\mathfrak{n} ; \boldsymbol{\theta}]$ is the noise-free part of Eq. (3) where all its parameters are defined by $\boldsymbol{\theta}$.

$L S(\boldsymbol{\theta})$ is multidimensional and nonlinear. Thus a direct minimization of Eq. 22 is difficult due to the high non-linearity of the function and the parameter number. Classical optimization techniques such as gradient descent, Gauss-Newton and EM algorithms do not ensure convergence to the global minimum when local minima are numerous. This problem can be overcome with meta-heuristic approaches. In the context of this paper, we proposed to use the Simulated Annealing technique [25] for its efficiency when a desired global extremum is hidden in many local extrema, and also because of its simple implementation.

\section{Application of the Proposed Approach over Simulated Data}

The aim of this section is to test the method proposed in terms of signal processing performance. Simulated vibration signals have been generated according to a physical model and Monte Carlo Simulations have provided the statistical properties of the result. Finally, the same signals have been processed manually in order to highlight the performance gain. The data are generated using a model of high-rise building defined as a continuous beam model. In this model, the mass of each storey of the building is considered to be mostly concentrated at its floor, and a lumped mass modeling is assumed for this structure. Therefore, the Duhamel integral gives the elastic motion of the building at each floor 
knowing only the mass of each floor, the modal parameters (namely the mode shapes, frequencies and damping ratios), and the ground motion [26].

Six different 50-story building models are simulated in order to reflect as possible a panel of high-rise buildings with different structural designs. The acceleration response of the top floor of each of the simulated buildings is computed and denoted $T o p_{1}$ to $T o p_{6}$ respectively. According to their different spectral structures, the six signals are categorized as: well-spaced, quasi-spaced, and closely-spaced frequency modes as shown in Tab. 1 Only the frequencies and the damping ratios are considered in this table as these two parameters keep the same characteristics as the impulse response of the considered physical model of Eq. 2. This is not the case of the amplitude and the damping ratio that have changing values due to the stacking and averaging process of the RDT.

Table 1: The six simulated signals denoted as $T o p_{1}, T_{o p_{2}}, T_{o p_{3}}, \mathrm{Top}_{4}, \mathrm{Top}_{5}$, and Top 6 , where $f$ indicates the simulated frequency in $(\mathrm{Hz})$ and $\xi$ the simulated damping ratio in (\%).

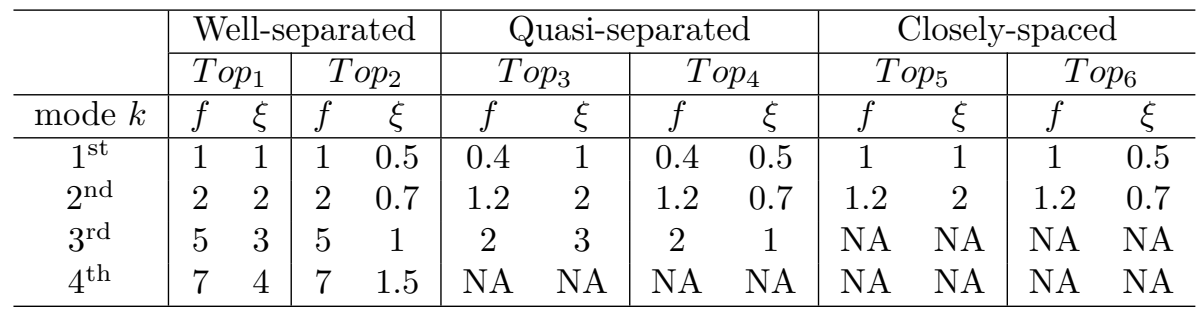

All the six simulated signals are generated with a length of 360,000 points, and a sampling frequency of $200 \mathrm{~Hz}$. The application of AMBA is tested under 3 different signal-to-noise ratio levels $(S N R), 0 \mathrm{~dB}, 9 \mathrm{~dB}$, and $15 \mathrm{~dB}$ and over 100 runs of the noise in order to proceed to Monte Carlo simulations.

The filter-free RDT is applied over the multi-component simulated signals in question. The estimated MRDS is then decimated to a Nyquist rate of $20 \mathrm{~Hz}$ to reduce the computation time. The triggering condition used in this section is the positive point type [27]. Indeed, due to the high content of noise we chose to use such a triggering condition, so that sufficient triggering points are available.

The length of the segments $N_{r d s}$ that are stacked and averaged in the RDT of all the simulated signals is chosen carefully based on Monte-Carlo simulations 
[28. For $T o p_{1}$ and $T o p_{2}$, the $N_{r d s}$ is set to be 5 periods for all the SNR levels. Similarly, for $T o p_{3}$ and $T o p_{4}$ except when the SNR is equal to $0 \mathrm{~dB}$, the $N_{r d s}$ was set to be equal to 7 periods. In the case of closely-spaced frequency modes, $T o p_{5}$ and $T o p_{6}$, the $N_{r d s}$ was set to 12 periods, this choice helps increasing the frequency resolution over the Welch spectrum, and thus allows identifying the closely-spaced frequency modes.

The statistical results provided by the Monte Carlo simulation are in tables 2. 3 and 4 . The accuracy of the estimation is quantified by the error $E$ as

$$
E=\frac{1}{D} \sum_{i=1}^{D} \frac{\left|x-\hat{x}_{i}\right|}{x},
$$

where $x$ describes either a simulated natural frequency or a damping ratio of the mode under study, $D$ is the number of the noise realizations, $\hat{x}_{i}$ is the estimated 300 value of $x \forall i \in[1, D]$.

Tables 2,3 and 4 show that the mean estimated frequencies of the six simulated signals are stable across all modes with a relative error of estimation $E$ less than $5 \%$. This error becomes noticeable $(E \leq 10 \%)$ only for the modes where the damping ratio is $3 \%<\xi \leq 4 \%$. The estimation is not deteriorated until the SNR reaches $0 \mathrm{~dB}$, where the error is then $10 \%<E \leq 30 \%$, and the noise variance is equal to the average signal power.

The errors of the mean values of the estimated damping ratios are in the range $10 \%<E \leq 15 \%$. The most significant outliers occur for the modes of high frequency with a damping ratio greater than or equal to $3 \%$. Given that the modes in high frequencies damp faster than those of their lower counterparts, this makes them indistinguishable from noise. Moreover, the amplitude of the RDS gets smaller at its end. As the amplitude for highly damped modes get smaller rapidly, then the estimation will be biased by this reduction and the error $E$ will be rather high $\leq 20 \%$. The error of the modes possessing both high damping and high frequency becomes higher when the noisy observation is generated with low SNR level $(E \leq 30 \%)$.

For the purpose of comparison without statistical purpose, the classical RDT 

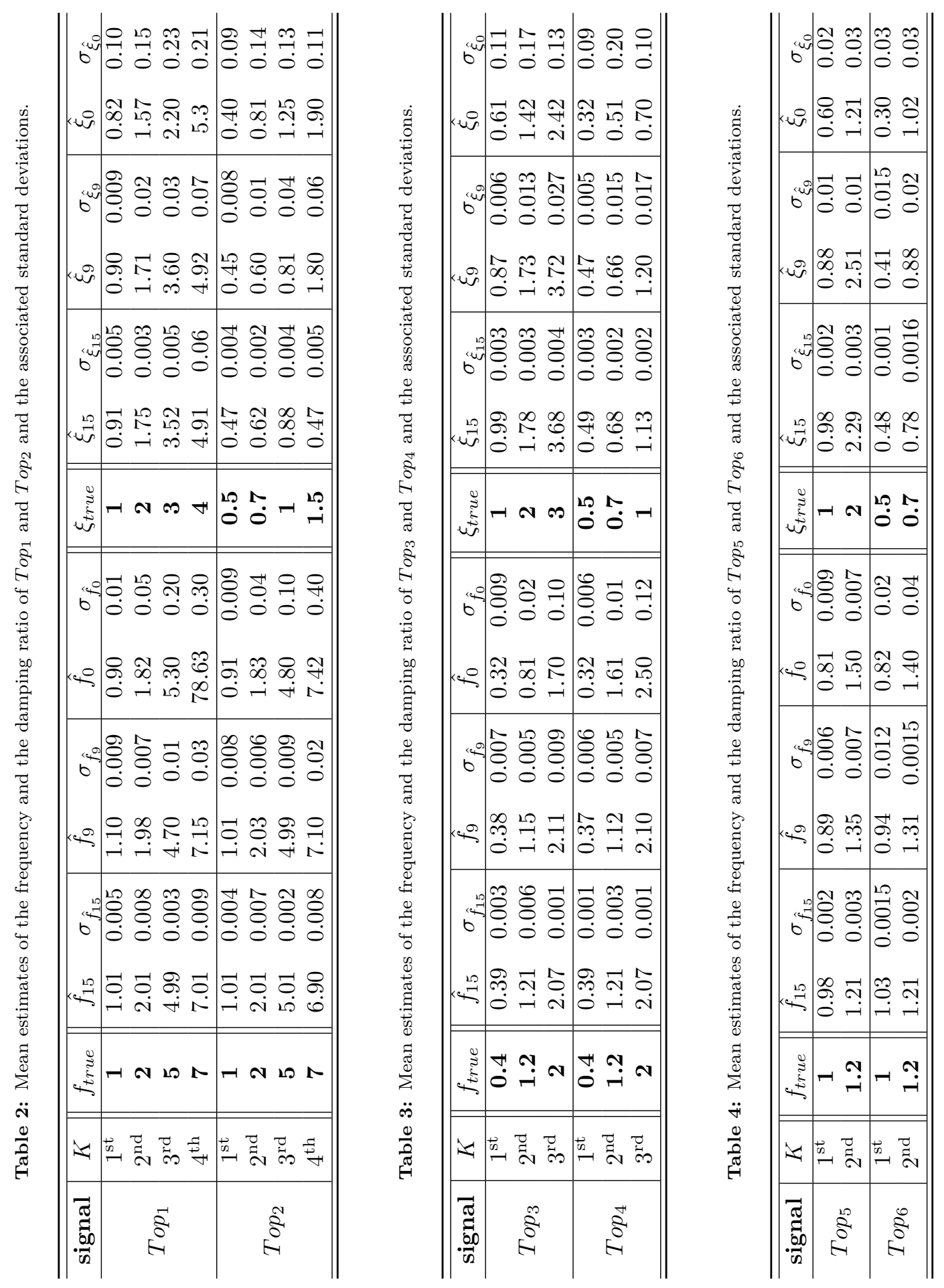
with a preliminary manual filtering process is applied over the six simulated signals. The Butterworth filter is used to filter each mode $k$.

The comparative results are shown in Fig. 6 and Fig. 7 in terms of an estimation error of the frequencies and the damping ratios respectively.

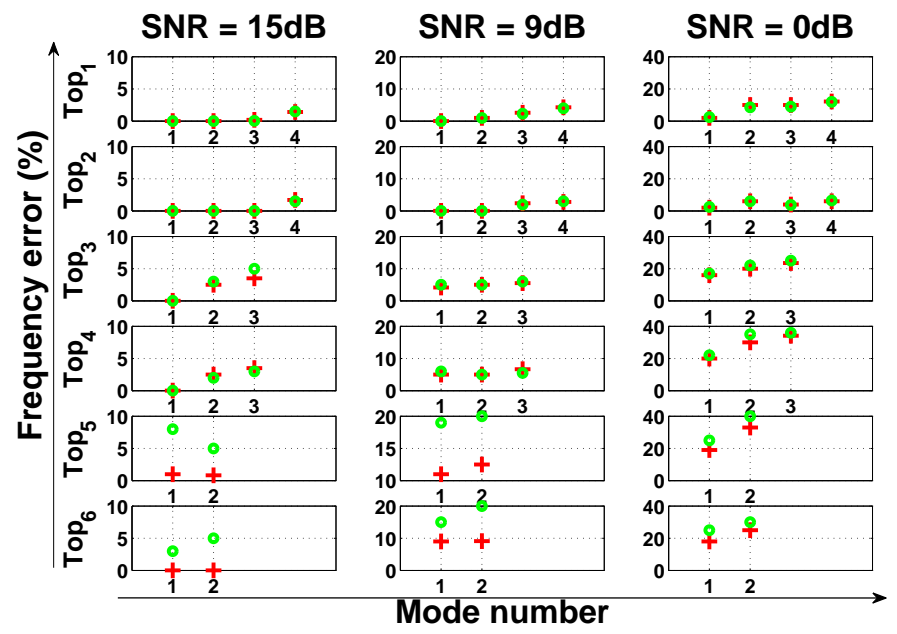

Figure 6: Estimated frequency using AMBA via a filter-free RDT (+) and an RDT pre-processed by filtering (o).

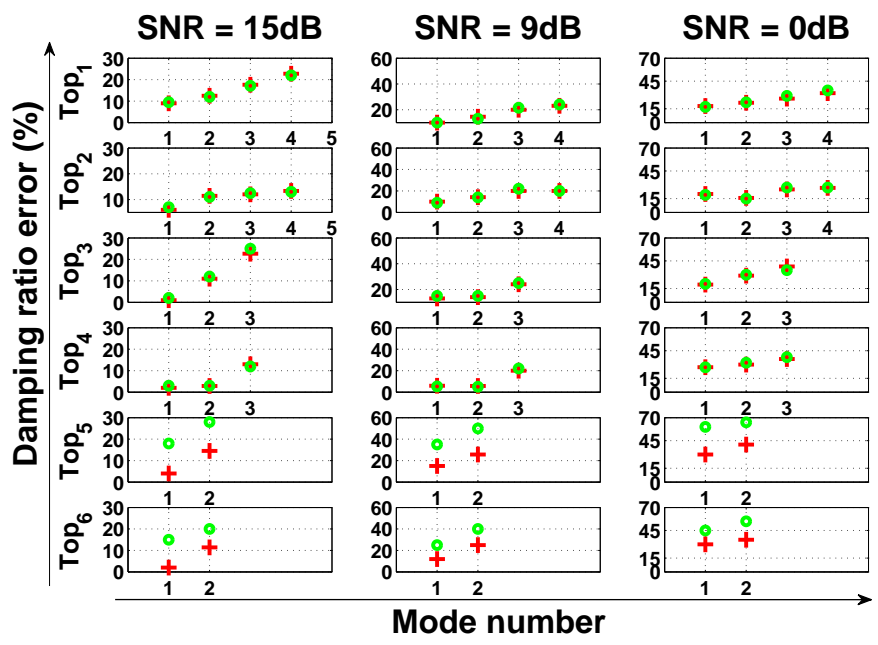

Figure 7: Estimated damping ratio using AMBA via a filter-free RDT $(+)$ and an RDT pre-processed by filtering (o).

The modal estimation of the signals of the first two categories in Tab. 1 
i.e., $T o p_{1}, T o p_{2}, T o p_{3}$ and $T o p_{4}$ where the modes are sufficiently spaced, exhibit nearly the same estimation results of frequency and damping ratio for both approaches, the filter-free RDT and the RDT pre-processed by a manual filtering. The frequency estimation error in Fig. 6 is less than 10\%, and that of the damping ratio in Fig. 7 is less than 20\%. The estimation results of the frequencies and the damping ratios were deteriorated when the SNR is $0 \mathrm{~dB}$, the error thus becomes noticeable $(E \geq 30 \%)$.

For the signals with closely-spaced frequency modes, i.e., Top 5 , and $T o p_{6}$, the accuracy of AMBA with the filter-free RDT is higher than that of the RDT pre-processed by a manual filtering. The error of the frequency estimation in AMBA presents more than $20 \%$ gain as compared to the manual method. The error of the damping ratio estimation is improved by $15 \%$ as well.

This section shows the interest of AMBA. It shows its importance for the cases where filtering becomes very difficult like the case of closely-spaced modes with high additive noises. Moreover the performances are hold for the other cases.

\section{Conclusions}

This paper proposed a new automatic modal analysis approach for multicomponent signals of high-rise buildings subjected to ambient vibrations. This approach is called an Automatic Model-Based Approach (AMBA).

AMBA starts by applying the Random Decrement Technique (RDT) directly over the multi-component signal to extract a free-response equivalent to the system impulse response. Such an application skips the preliminary filtering process prior to the RDT analysis, and thus is named a filter-free RDT. It leads to the estimation of a Multi-mode Random Decrement Signature, referred to as an MRDS.

In a second stage of AMBA, a non-parametric spectral-based method is then proposed to estimate the modal parameters of the MRDS. This stage relies on a global spectrum estimation of the MRDS to keep the best frequency resolution. 
Over the estimated spectrum, an automatic peak-detection method is applied to extract the peaks from the noise spectrum that is estimated by a multi-pass filtering. A modal estimate is then achieved for each of the detected modes.

However, the spectral-based method is limited to the resolution of the Fourier spectrum. To solve such a limitation, the modal parameters of the MRDS are then refined in a final stage using a parametric maximum likelihood strategy.

Considering all the above, AMBA is applicable over a single-channel record, has no parameters to be set, and no manual initialization phase. It is not restricted to SDOF systems, i.e., it deals with multi-mode ambient vibration signals, and takes into consideration the estimation of the damping ratio, and the closely-spaced frequency modes. AMBA is capable of distinguishing the true modes from the noisy ones thanks to an automatic peak-detection method which avoids any user interaction.

The application of AMBA has been assessed over simulated data generated using a continuous beam model excited by a Gaussian white noise. The results obtained from 100 runs have been analyzed in order to assess the application of AMBA in terms of robustness, accuracy and precision. Encouraging results have been obtained despite low SNR levels and different signal categories, in particular the possibility to estimate damping ratios in a robust and automatic way.

Further validations of AMBA over real-world ambient vibration signals are in progress. Also some investigations should be held to assess the performance of AMBA in view of continuous and long-term structural health monitoring 375 applications.

\section{Acknowledgements}

This work has been supported by French Research National Agency (ANR) through RISKNAT program (project URBASIS ANR-09-RISK-009). 


\section{References}

[1] P. Verboven, E. Parloo, P. Guillaume, M. Van Overmeire, Autonomous structural health monitoring, part i: modal parameter estimation and tracking, Mechanical Systems and Signal Processing 16 (4) (2002) 637-657.

[2] S. Vanlanduit, P. Verboven, P. Guillaume, J. Schoukens, An automatic frequency domain modal parameter estimation algorithm, Journal of Sound and Vibration 265 (3) (2003) 647-661.

[3] B. Peeters, G. De Roeck, One-year monitoring of the Z 24-Bridge: environmental effects versus damage events, Earthquake engineering \& structural dynamics 30 (2) (2001) 149-171.

[4] J. M. W. Brownjohn, E. P. Carden, Tracking the effects of changing environmental conditions on the modal parameters of Tamar Bridge, in: Proceedings of The 3rd international conference on structural health monitoring and intelligent infrastructure, 2007.

[5] A. Deraemaeker, E. Reynders, G. De Roeck, J. Kullaa, Vibration-based structural health monitoring using output-only measurements under changing environment, Mechanical systems and signal processing 22 (1) (2008) $34-56$.

[6] P. Andersen, R. Brincker, M. Goursat, L. Mevel, Automated modal parameter estimation for operational modal analysis of large systems, in: Proceedings of The 2nd International Operational Modal Analysis Conference (IOMAC), 2007, pp. 299-308.

[7] F. Magalhães, A. Cunha, E. Caetano, Permanent monitoring of Infante D. Henrique bridge based on FDD and SSI-COV methods, in: Proceedings of ISMA, International Conference on Noise and Vibration Engineering, Leuven, Belgium, 2008.

[8] F. Magalhães, Á. Cunha, E. Caetano, Dynamic monitoring of a long span arch bridge, Engineering Structures 30 (11) (2008) 3034-3044. 
[9] F. Magalhaes, A. Cunha, E. Caetano, Online automatic identification of the modal parameters of a long span arch bridge, Mechanical Systems and Signal Processing 23 (2) (2009) 316-329.

[15] T.-P. Le, P. Paultre, Modal identification based on continuous wavelet transform and ambient excitation tests, Journal of Sound and Vibration 331 (9) (2012) 2023-2037.

[16] A. Mikael, P. Gueguen, P.-Y. Bard, P. Roux, M. Langlais, The analysis 430 of long-term frequency and damping wandering in buildings using the random decrement technique, Bulletin of the Seismological Society of America 103 (1) (2013) 236-246.

[17] P. Gueguen, M. Langlais, P. Roux, J. Schinkmann, I. Douste-Bacqué, Frequency and Damping Wandering in Existing Buildings Using the Random 
Decrement Technique, in: EWSHM-7th European Workshop on Structural Health Monitoring, 2014.

[18] H. A. Cole, On-The-Line Analysis of Random Vibrations, AIAA Paper (1968) 68-288.

[19] S. R. Ibrahim, Random decrement technique for modal identification of structures, Journal of Spacecraft and Rockets 14 (11) (1977) 696-700.

[20] J. K. Vandiver, A. B. Dunwoody, R. B. Campbell, M. F. Cook, A mathematical basis for the random decrement vibration signature analysis technique, Journal of Mechanical Design 104 (2) (1982) 307-313.

[21] C. Michel, Vulnérabilité Sismique de l'échelle du bâtiment à celle de la villeApport des techniques expérimentales in situ-application à grenoble, Ph.D. thesis, Université Joseph-Fourier-Grenoble I, France (In french) (2007).

[22] M. Durnerin, Une stratégie pour l'interprétation en analyse spectrale. Détection et caractérisation des composantes d'un spectre., Ph.D. thesis, Institut National Polytechnique de Grenoble-INPG, France (In French) (1999).

[23] C. Mailhes, N. Martin, K. Sahli, G. Lejeune, A Spectral Identiy Card, in: EUropean SIgnal Processing Conference, EUSIPCO 06, 2006.

[24] Z. Li, N. Martin, M. Vieira, P. Guéguen, Parameter estimation of shorttime multi-component signals using damped-amplitude \& polynomialfrequency model, in: Statistical Signal Processing Workshop (SSP), 2011 IEEE, IEEE, 2011, pp. 545-548.

[25] S. Kirkpatrick, Optimization by simulated annealing: Quantitative studies, Journal of statistical physics 34 (5-6) (1984) 975-986.

[26] M. Perrault, P. Guéguen, A. Aldea, D. Sorin, Reducing the uncertainties of the fragility curves using experimental testing in existing buildings: the 
case of the BRD Tower of Buccarest (Romania), Earthquake Engineering and Engineering Vibration 12 (4).

[27] J. C. Asmussen, Modal analysis based on the random decrement technique: application to civil engineering structures, Ph.D. thesis, unknown (1997).

465

[28] F. Nasser, Automatic modal variation tracking via a filter-free random decrement technique, Application to ambient vibration recordings on highrise buildings., Ph.D. thesis, Institut National Polytechnique de GrenobleINPG (2015). 